\title{
Inhibitory effects of delphinidin on the proliferation of ovarian cancer cells via PI3K/AKT and ERK 1/2 MAPK signal transduction
}

\author{
WHASUN LIM ${ }^{1,2}$ and GWONHWA SONG ${ }^{1,3}$ \\ ${ }^{1}$ Department of Biotechnology, College of Life Sciences and Biotechnology, Korea University, Seoul 02841; \\ ${ }^{2}$ Department of Biomedical Sciences, Catholic Kwandong University, Gangneung, Gangwon-do 25601; \\ ${ }^{3}$ Institute of Animal Molecular Biotechnology, Korea University, Seoul 02841, Republic of Korea
}

Received February 25, 2016; Accepted March 7, 2017

DOI: $10.3892 / 01.2017 .6232$

\begin{abstract}
Delphinidin is a member of the anthocyanidin family and is a natural pigment in red cabbage, berries, sweet potatoes and grapes. It possesses nutraceutical properties against various chronic diseases and types of cancer. However, little is known about its preventative effects on epithelial ovarian cancer, a disease that is associated with a low survival rate, a poor prognosis and a high rate of recurrence. The results of the present study demonstrated that the proliferation of SKOV3 cells decreased in a dose-dependent manner in response to treatment with delphinidin, and the phosphorylation of carcinogenic protein kinases associated with the progression of epithelial ovarian cancer was affected by delphinidin treatment. The levels of phosphorylated protein kinase B (AKT), ribosomal protein S6 kinase $\beta$-1 (P70S6K), ribosomal protein $\mathrm{S}$ (S6), extracellular signal-regulated kinase (ERK)1/2 and p38 were suppressed by increasing concentrations of delphinidin. Furthermore, the combination of certain pharmacological inhibitors, including phosphoinositide 3-kinase (PI3K; LY294002), ERK1/2 (U0126) and delphinidin significantly reduced the proliferation of SKOV3 cells and the phosphorylation of each of those target proteins. In addition, delphinidin treatment exerted anti-proliferative effects on paclitaxel-resistant SKOV3 cells, compared with treatment with paclitaxel alone. These results indicate that delphinidin inhibits the proliferation of SKOV3 cells through inactivation of PI3K/AKT and ERK1/2 mitogen-activated protein kinase signaling cascades, and that this cell signaling pathway may be a pivotal therapeutic target for the prevention of epithelial ovarian cancer, including paclitaxel-resistant ovarian cancer.
\end{abstract}

Correspondence to: Professor Gwonhwa Song, Department of Biotechnology, College of Life Sciences and Biotechnology, Korea University, 145 Anam-ro, Seoul 02841, Republic of Korea

E-mail: ghsong@korea.ac.kr

Key words: delphinidin, cell proliferation, ovarian cancer, signal transduction, chemotherapy

\section{Introduction}

Flavonoids are the most abundant polyphenols in the human diet and may be subdivided into anthocyanidins, flavanols, flavanones, flavonols, flavones and isoflavones, according to structural and functional features (1). In a previous review, dietary flavonoids were highlighted for their beneficial biological activities in the improvement of human health against osteoporosis, cancer, cardiovascular diseases, neurodegenerative diseases and diabetes in a variety of epidemiological studies (2). As a subfamily of flavonoids, anthocyanins are important plant pigments with a red, blue or purple color; they are principally identified in berries, pomegranates, red cabbage, grapes, sweet potatoes and red wine (3). Although $>400$ anthocyanins have been identified in nature, only six anthocyanidins, including cyanidin, pelargonidin, peonidin, delphinidin, petunidin and malvidin, are ubiquitously distributed (4). Amongst anthocyanidins, delphinidin is known to have effective biological functions in the prevention of oxidative stress, inflammation, angiogenesis, metastasis and carcinogenesis (5-7). An increasing number of studies have identified the potential protective effects of delphinidin against carcinogenesis of the breast (8), prostate (9), lungs (10), liver (11) and colon (12) and against fibrosarcoma (13) by regulating cell signal transduction. However, little is known about the functional role of delphinidin in the female reproductive system and gynecologic types of cancer, including of the cervix, ovary, endometrium, vagina and vulva $(14,15)$.

Gynecological cancer subtypes are malignant carcinomas of the female reproductive tract, the most common of which is epithelial ovarian cancer (EOC); EOC is the fifth leading cause of cancer-associated mortalities in women, due to a lack of symptoms and effective biomarkers for diagnosis in the early stages (16). The current first-line treatment strategy for EOC is intensive surgical cytoreduction, followed by systemic chemotherapy with a platinum- or taxane-based regimen (17). However, advanced EOC [International Federation of Gynecological Oncology stages III-IV (18)] accounts for 75\% of total patients diagnosed with ovarian cancer with low (5-year) survival rates and a high recurrence rate of $\sim 80 \%$ with failure to respond to conventional chemotherapy (19). Thus, it is 
necessary to develop novel chemotherapeutic approaches to increase the susceptibility of EOC to novel treatments, in order to improve the median progression-free survival and overall survival times. Therefore, the present study determined the potential role of delphinidin in EOC through investigating the following: Effects of delphinidin on the viability of SKOV3 ovarian adenocarcinoma cells; cell signaling mechanisms regulated by delphinidin against the proliferation of SKOV3 cells; comparative chemotherapeutic effects between delphinidin and conventional chemotherapy drugs on the proliferation of SKOV3 cells. The results of the present study indicate that delphinidin exerts anti-proliferative effects in SKOV3 cells via inactivation of the phosphoinositide- 3 kinase (PI3K) and extracellular-regulated kinase (ERK)1/2 mitogen-activated protein kinase (MAPK) signaling pathways. Therefore, this may be an effective synergistic regimen for the enhancement of conventional chemotherapy for patients with EOC.

\section{Materials and methods}

Chemicals. Delphinidin was purchased from Indofine Chemical Company, Inc. (Hillsborough, NJ, USA). SB203580 and U0126 were purchased from Enzo Life Sciences, Inc. (Farmingdale, NY, USA) and LY294002 was obtained from Cell Signaling Technology, Inc. (Danvers, MA, USA). The antibodies against phosphorylated-AKT [serine $(\mathrm{Ser})^{473}$ ] (catalog no. 4060), ERK1/2 [threonine (Thr) ${ }^{202} / \mathrm{Tyr}^{204}$, (catalog no. 9101; Cell Signaling Technology, Inc.)] c-Jun N-terminal kinase (JNK; Thr ${ }^{183} / \mathrm{Thr}^{185,}$ catalog no. 4668), p38 $\left(\mathrm{Thr}^{180} / \mathrm{Thr}^{182}\right.$; catalog no. 4511), ribosomal protein S6 kinase $\beta-1$ (P70S6K; $\mathrm{Thr}^{421} / \mathrm{Ser}^{424}$; catalog no. 9204) and S6 (Ser ${ }^{235 / 236}$, catalog no. 2211), and total AKT (catalog no. 9272), ERK1/2 (catalog no.4695), JNK (catalog no. 9252), p38 (catalog no. 9212), P70S6K (catalog no. 9202) and S6 (catalog no. 2217) were purchased from Cell Signaling Technology, Inc. All antibodies were diluted to 1:1,000 for western blot analysis. Cisplatin and paclitaxel were purchased from Sigma-Aldrich (Merck KGaA, Darmstadt, Germany).

Cell culture. SKOV3 cells were purchased from the American Type Culture Collection (Manassas, VA, USA) and maintained in McCoy's 5A (modified) medium (cat. no. 16600-082; Invitrogen; Thermo Fisher Scientific, Inc., Waltham, MA, USA) supplemented with $10 \%$ fetal bovine serum (Hyclone; GE Healthcare Life Sciences, Logan, UT, USA) at $37^{\circ} \mathrm{C}$ in an atmosphere containing $\mathrm{CO}_{2}$.

Proliferation assay. Proliferation assays were conducted using a Cell Proliferation ELISA, 5-bromo-2'-deoxyuridine (BrdU) kit (catalog no. 11647229001; Roche Diagnostics, Indianapolis, IN, USA), according to the manufacturer's protocol. Briefly, SKOV3 cells $\left(3 \times 10^{3}\right.$ cells $\left./ 100 \mu \mathrm{l}\right)$ were seeded onto a 96 -well plate and incubated at $37^{\circ} \mathrm{C}$ for $24 \mathrm{~h}$ in serum-free McCoy's 5A (modified) medium. Cells were then treated with $10 \mu \mathrm{M}$ delphinidin alone or with various inhibitors of cell signaling proteins including $20 \mu \mathrm{M}$ LY294002, $20 \mu \mathrm{M} \mathrm{SB} 203580$ and $10 \mu \mathrm{M}$ U0126 or conventional chemotherapy including $20 \mu \mathrm{M}$ paclitaxel and $50 \mu \mathrm{M}$ cisplatin in a final volume of $100 \mu \mathrm{l} /$ well. Following $48 \mathrm{~h}$ of incubation at $37^{\circ} \mathrm{C}, 10 \mu \mathrm{M}$ BrdU was added to the cell culture and the cells were incubated for an additional
$2 \mathrm{~h}$ at $37^{\circ} \mathrm{C}$. After labeling cells with BrdU, the fixed cells were incubated with anti-BrdU-peroxidase (POD) working solution for $90 \mathrm{~min}$ at room temperature. The anti-BrdU-POD binds to BrdU incorporated in newly synthesized cellular DNA, and these immune complexes were detected by the reaction to 3,3',5,5'-tetramethylbenzidine substrate. The absorbance values of the reaction product were quantified by measuring the absorbance at 370 and $492 \mathrm{~nm}$ using an ELISA reader. These experiments were performed in triplicate.

TUNEL assay. The SKOV3 cells $\left(3 \times 10^{4}\right.$ cells/300 $\left.\mu \mathrm{l}\right)$ were seeded onto confocal dishes (catalog no. 100350; SPL Life Sciences Co., Ltd., Pocheon, Korea) and then incubated for $24 \mathrm{~h}$ at $37^{\circ} \mathrm{C}$ in serum-free McCoy's 5A. The cells were subsequently treated with delphinidin at a final concentration of $10 \mu \mathrm{M}$ in $10 \mathrm{ml}$ for $48 \mathrm{~h}$ at $37^{\circ} \mathrm{C}$ in a $\mathrm{CO}_{2}$ incubator. Following incubation, the cells were subjected to TUNEL staining using an in situ Cell Death Detection kit, TMR red (Roche Diagnostics) according to the manufacturer's protocol. Fluorescence was detected using an LSM 710 (Carl Zeiss AG, Oberkochen, Germany) confocal microscope at magnifications 40x and 80x fitted with a digital microscope AxioCam camera and using Zen 2009 software (Carl Zeiss AG). These experiments were performed in triplicate.

Western blot analysis. The concentration of proteins from whole-cell extracts was determined using a Bradford protein assay (Bio-Rad Laboratories, Inc., Hercules, CA, USA) with bovine serum albumin (Sigma-Aldrich; Merck KGaA) as the standard. Proteins were denatured, separated using 10\% SDS-PAGE and transferred to nitrocellulose membranes. The membranes were blocked using $5 \%$ bovine serum albumin in TBST buffer for $1 \mathrm{~h}$ at room temperature. Immunoreactive proteins were detected using goat anti-rabbit polyclonal antibodies against phosphorylated proteins and total proteins at a dilution of 1:1,000 (as mentioned in the previous section). The blots were developed using enhanced chemiluminescence detection (SuperSignal ${ }^{\mathrm{TM}}$ West Pico; Pierce; Thermo Fisher Scientific, Inc.) and quantified by measuring the intensity of light emitted from correctly sized bands under ultraviolet light, using a ChemiDoc ${ }^{\mathrm{TM}}$ EQ system and Quantity One ${ }^{\circledR}$ software (version 4.5; Bio-Rad Laboratories, Inc.). Total protein normalization was used to quantify the abundance of the phosopho-proteins. As a loading control, total proteins were used to normalize the results from the detection of proteins by western blotting. Multiple exposures of each western blot were performed to ensure linearity of the chemiluminescent signals. These experiments were performed in triplicate.

Determination of apoptosis by Annexin $V$ and propidium iodide (PI) staining. Apoptosis of SKOV3 cells induced by delphinidin was analyzed using a fluorescein isothiocyanate (FITC)-Annexin V apoptosis detection kit I (BD Biosciences, Franklin Lakes, NJ, USA). The cells ( $4 \times 10^{5}$ cells) were seeded onto 6-well plates and incubated for $24 \mathrm{~h}$ at $37^{\circ} \mathrm{C}$ in a $\mathrm{CO}_{2}$ incubator in serum-free medium until the cells reached $70-80 \%$ confluence. The cells were then treated separately with $10 \mu \mathrm{M}$ delphinidin, $20 \mu \mathrm{M}$ LY294002, $10 \mu \mathrm{M}$ U0126 or a combination of $10 \mu \mathrm{M}$ delphinidin with each inhibitor for $48 \mathrm{~h}$ at $37^{\circ} \mathrm{C}$ in a $\mathrm{CO}_{2}$ incubator. Supernatants were removed from the 
A

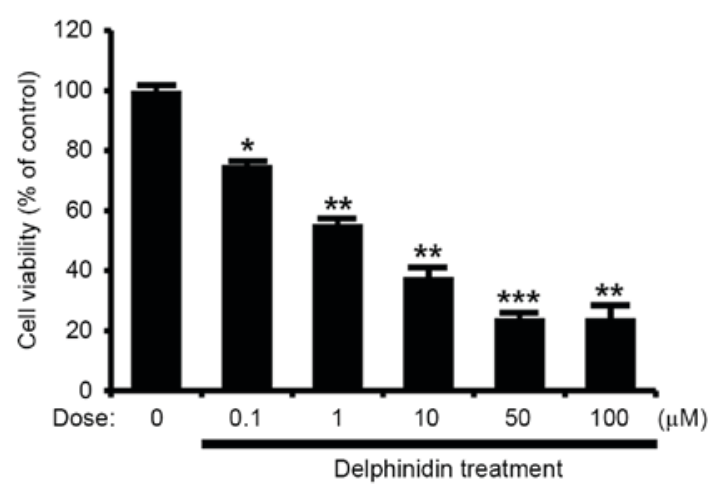

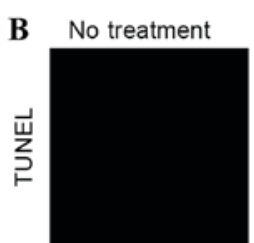
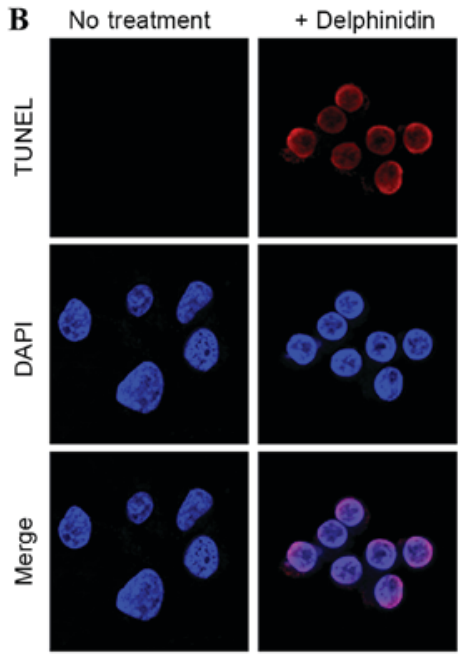

Figure 1. Effects of delphinidin on the viability of SKOV3 ovarian adenocarcinoma cells. (A) Dose-response effects of delphinidin on the proliferation of SKOV3 cells were analyzed and are presented as a percentage relative to the non-treated controls (100\%). (B) In situ cell death was determined in SKOV3 cells following delphinidin treatment using TUNEL technology, which labels DNA strand breaks with red fluorescence. The nuclei of SKOV3 cells were counterstained with DAPI (blue). ${ }^{* * *} \mathrm{P}<0.001 ;{ }^{* * *} \mathrm{P}<0.01 ;{ }^{*} \mathrm{P}<0.05$, statistically significant differences by treatment. TUNEL, Terminal deoxynucleotidyl transferase dUTP nick end labeling.

culture dishes using a $10 \mathrm{ml}$ serological pipette, and adherent cells were detached using $0.25 \%$ trypsin-EDTA. The cells were collected by centrifugation $(300 \mathrm{x} \mathrm{g})$ at room temperature for $5 \mathrm{~min}$, washed with PBS and resuspended in 1X binding buffer (BD Biosciences). The cell suspension (100 $\mu \mathrm{l})$ was then transferred into a $5 \mathrm{ml}$ culture tube and incubated with $5 \mu \mathrm{l}$ FITC-Annexin V and $5 \mu \mathrm{l}$ PI for $15 \mathrm{~min}$ at room temperature in the dark. Subsequently, $400 \mu 11 \mathrm{X}$ binding buffer was added to the $5 \mathrm{ml}$ culture tube. Fluorescence intensity was analyzed using FACSCalibur ${ }^{\mathrm{TM}}$ at $488 \mathrm{~nm}$ (BD Biosciences).

Cell cycle assay. SKOV3 cells ( $3 \times 10^{5}$ cells) were seeded onto 6 -well plates and incubated for $24 \mathrm{~h}$ in serum-free medium at $37^{\circ} \mathrm{C}$ until the cells reached $70-80 \%$ confluence. The cells were then treated separately with $10 \mu \mathrm{M}$ delphinidin, $20 \mu \mathrm{M}$ LY294002, $10 \mu \mathrm{M}$ U0126 or a combination of $10 \mu \mathrm{M}$ delphinidin with each inhibitor for $48 \mathrm{~h}$ at $37^{\circ} \mathrm{C}$ in an incubator containing $\mathrm{CO}_{2}$. Supernatants were transferred from the culture dishes into collecting tubes and adherent cells were detached using $0.25 \%$ trypsin-EDTA. The cells were collected via centrifugation $(300 \mathrm{x} \mathrm{g})$ at room temperature for $5 \mathrm{~min}$, washed twice with cold PBS and fixed in chilled-70\% ethanol at $4^{\circ} \mathrm{C}$ overnight. Cells were subsequently washed twice with PBS, incubated with $10 \mu \mathrm{g} / \mathrm{ml}$ RNase A (Sigma-Aldrich; Merck KGaA) and $50 \mu \mathrm{g} / \mathrm{ml}$ PI (BD Biosciences) in PBS at room temperature for $30 \mathrm{~min}$ in the dark and subjected to flow cytometry (FACSCalibur ${ }^{\mathrm{TM}}$; BD Biosciences).

Statistical analysis. Data obtained from the proliferation and Annexin V and PI assays were subjected to two-way analysis of variance according to the general linear model (PROC-GLM) of the SAS program (SAS Institute, Cary, NC, USA) in order to detect the effects of treatments on SKOV3 cells proliferation. Data are presented as least square means with standard error of the mean, unless otherwise stated. $\mathrm{P}<0.05$ was considered to indicate a statistically significant difference.

\section{Results}

Inhibitory effects of delphinidin on the viability of SKOV3 cells. The current study aimed to determine the effects of delphinidin on SKOV3 cells derived from ovarian adenocarcinoma. The results demonstrated a dose-dependent effect of delphinidin to inhibit the proliferation of SKOV3 cells between concentrations of $0,0.1,1,10,50$ and $100 \mu \mathrm{M}$ (Fig. 1A). Based on these results, delphinidin was used at a concentration of $10 \mu \mathrm{M}$ in further experiments, in order to determine its effects on the apoptosis of SKOV3 cells. As indicated by the TUNEL assay, apoptosis was induced in SKOV3 cells by nuclear DNA fragmentation in response to delphinidin treatment (Fig. 1B). In addition, the dose-dependent effects of delphinidin on the PI3K/AKT and MAPK signaling pathways were investigated (Fig. 2). Compared with basal values, delphinidin decreased the phosphorylation of PI3K downstream proteins, including AKT, P70S6K and S6, in a dose-dependent manner. In addition, the phosphorylated MAPK signaling proteins, including ERK1/2 MAPK and P38 MAPK, were downregulated with increasing concentrations of delphinidin, whereas JNK phosphorylation was not affected in SKOV3 cells treated with delphinidin. These results indicate that delphinidin exhibits anti-proliferative effects on SKOV3 cells and inhibits phosphorylation of the PI3K/AKT, ERK1/2 MAPK and P38 MAPK cell signaling proteins.

Effects of the inhibition of PI3K/AKT and MAPK signal transduction on SKOV3 cell proliferation. It was examined whether the delphinidin-suppressed proliferation of SKOV3 cells is associated with the PI3K/AKT, ERK1/2 MAPK and P38 MAPK signal transduction pathways. Cell proliferation assays were conducted in the absence or presence of delphinidin (10 $\mu \mathrm{M})$, LY294002 (20 $\mu \mathrm{M}$; PI3K inhibitor), SB203580 (20 $\mu \mathrm{M}$; P38 MAPK inhibitor) and U0126 (10 $\mu \mathrm{M}$; ERK1/2 MAPK inhibitor; Fig. 3A). Compared with non-treated SKOV3 
A
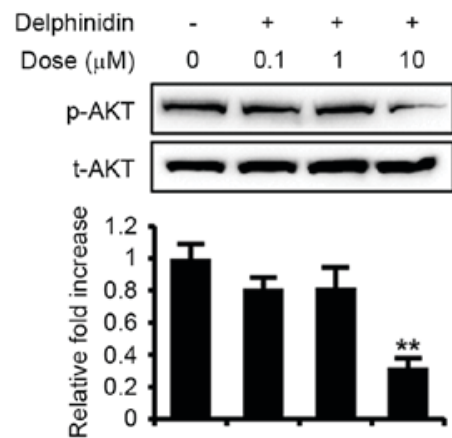

D
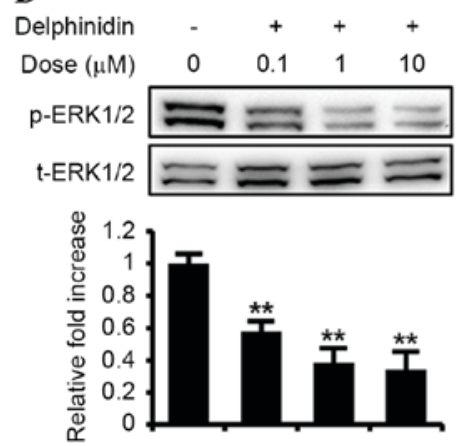

B
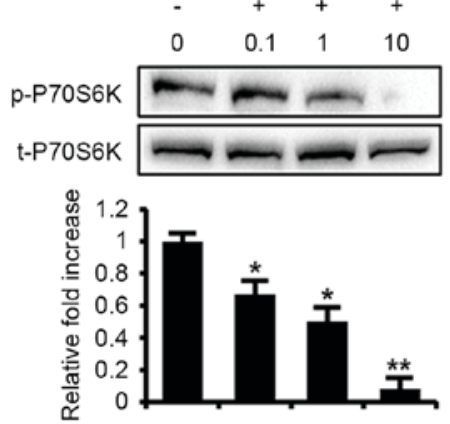

$\mathbf{E}$
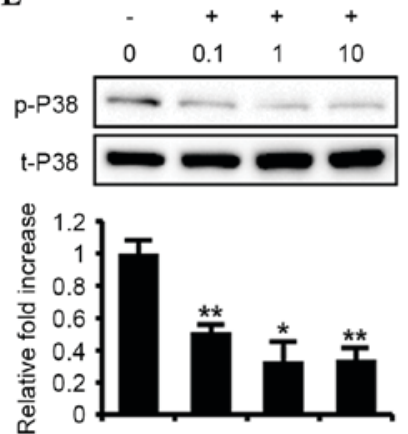

C

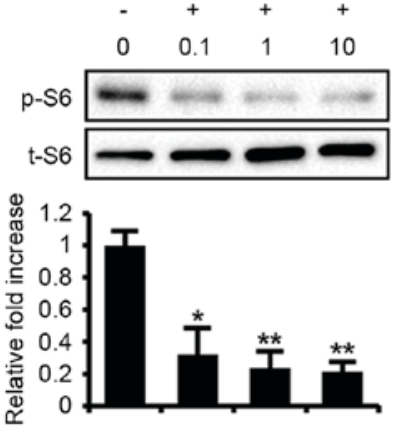

F
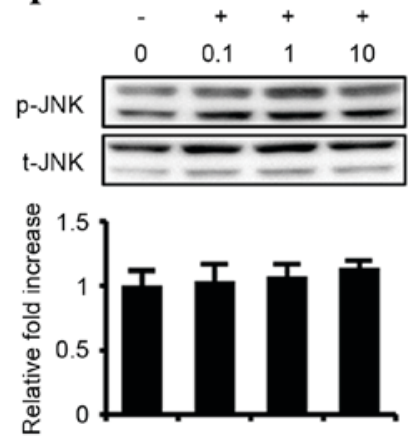

Figure 2. Dose-response effects of delphinidin on the phosphorylation of target signaling proteins in SKOV3 cells. Serum-starved SKOV3 cells were treated with delphinidin $(0,0.1,1$ and $10 \mu \mathrm{M})$ for $30 \mathrm{~min}$. Phosphorylation of (A) AKT, (B) P70S6K, (C) S6, (D) ERK1/2, (E) p38 and (F) JNK was estimated in SKOV3 cells incubated with delphinidin using western blot analysis. Blotting membranes were imaged to analyze the normalized values by calculating the abundance of phosphorylated protein relative to the total protein. ${ }^{* *} \mathrm{P}<0.01$; " $\mathrm{P}<0.05$. ERK, extracellular-regulated kinase; JNK, c-Jun $\mathrm{N}$-terminal kinase; $\mathrm{p}$, phosphorylated; t, total protein; AKT, protein kinase B.

A

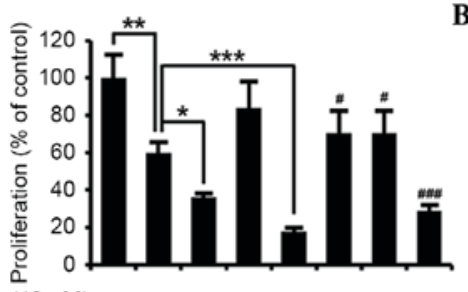

Delphinidin $(10 \mu \mathrm{M}) \cdot++++\quad-\quad$ -

$\mathrm{LY} 294002(20 \mu \mathrm{M})-{ }^{-}+-\quad+\quad-$

$\mathrm{SB} 203580(20 \mu \mathrm{M}) \cdot-\ldots+\ldots+.+$

$\mathrm{U} 0126(10 \mu \mathrm{M}) \cdot-\cdot+\cdots+$

C
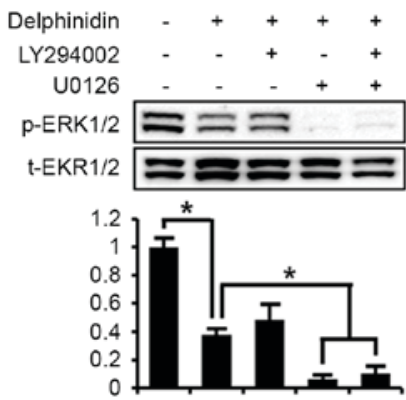

B

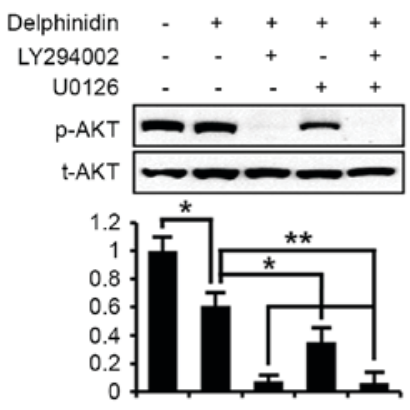

E

Delphinidin

LY294002
U0126

p-P70S6K $=-=$

t-P70S6K

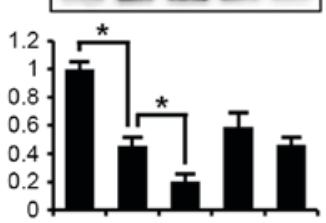

Delphinidin

LY294002

U0126
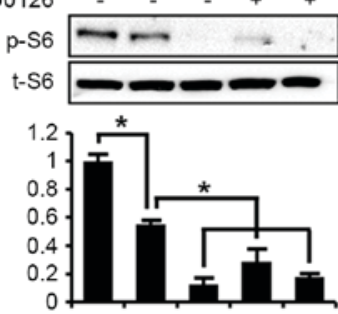

Figure 3. Delphinidin suppresses the proliferation of SKOV3 cells through the PI3K/AKT and ERK1/2 MAPK signaling pathways. (A) Effects of pharmacological inhibitors, including $20 \mu \mathrm{M}$ LY294002 (a PI3K inhibitor), $20 \mu \mathrm{M}$ SB203580 (a P38 MAPK inhibitor) or $10 \mu \mathrm{M}$ U0126 (an ERK1/2 MAPK inhibitor), of cell signaling pathways on the proliferation of SKOV3 cells treated with delphinidin. The results exhibit relative differences in cell proliferation, as compared with non-treated SKOV3 cells set at 100\%. Starved SKOV3 cells were pretreated with $20 \mu \mathrm{M}$ LY294002 or $10 \mu \mathrm{M}$ U0126, or with a combination of both inhibitors for $1 \mathrm{~h}$ and then incubated with $10 \mu \mathrm{M}$ delphinidin for $30 \mathrm{~min}$. Phosphorylation of (B) AKT, (C) ERK1/2, (D) P70S6K and (E) S6 was determined in SKOV3 cells by western blot analysis. Blotting membranes were imaged to analyze the normalized values by calculation of the abundance of phosphorylated protein relative to total protein. All quantitative data are presented as least square means with overall standard error of the mean. ${ }^{* * *} \mathrm{P}<0.001$; ${ }^{* *} \mathrm{P}<0.01$; ${ }^{*} \mathrm{P}<0.05$, compared with delphinidin treatment.. PI3K, phosphoinositide-3 kinase; ERK, extracellular-regulated kinase; MAPK, mitogen-activated protein kinase; $\mathrm{p}$, phosphorylated; $\mathrm{t}$, total protein; AKT, protein kinase B. 
A

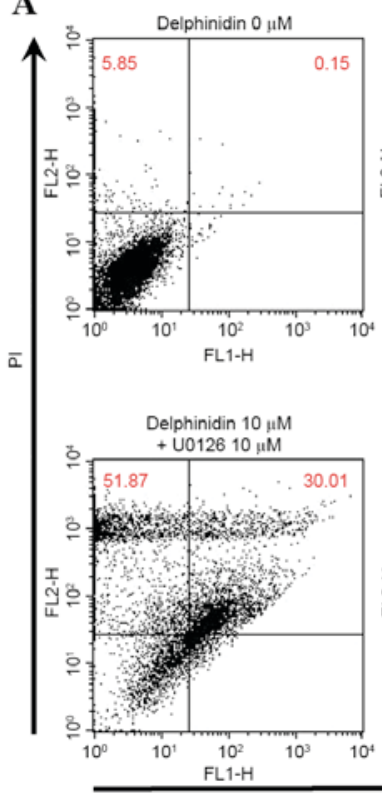

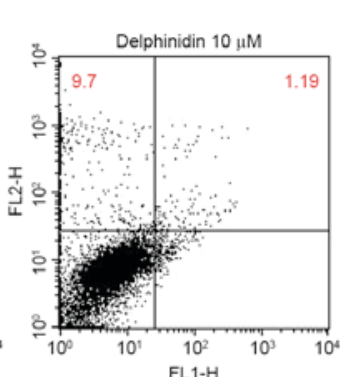
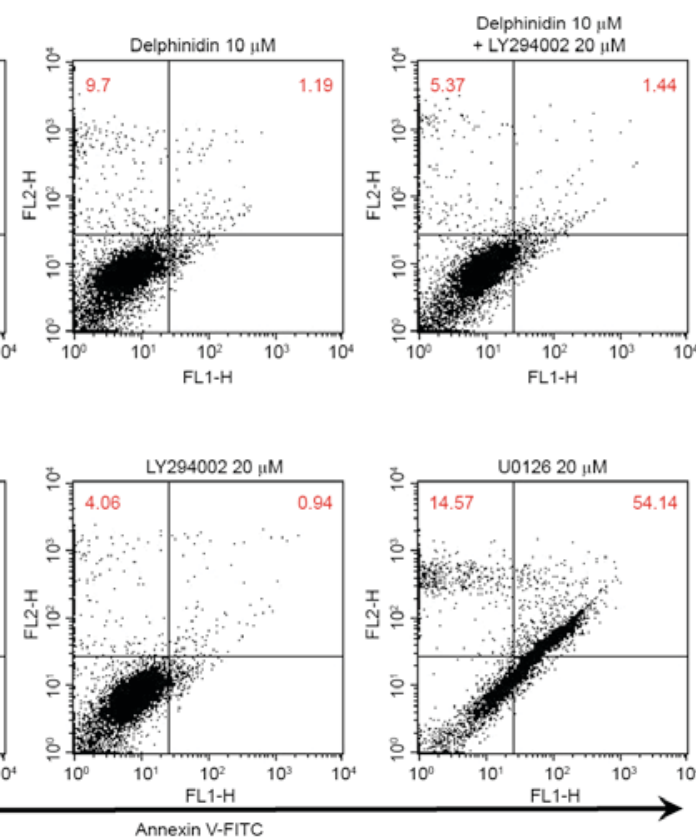

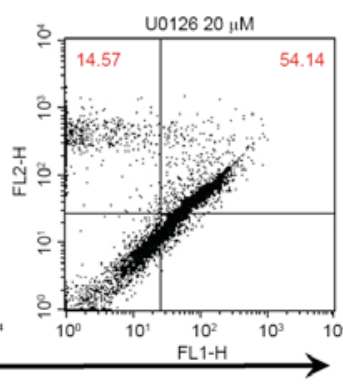

B
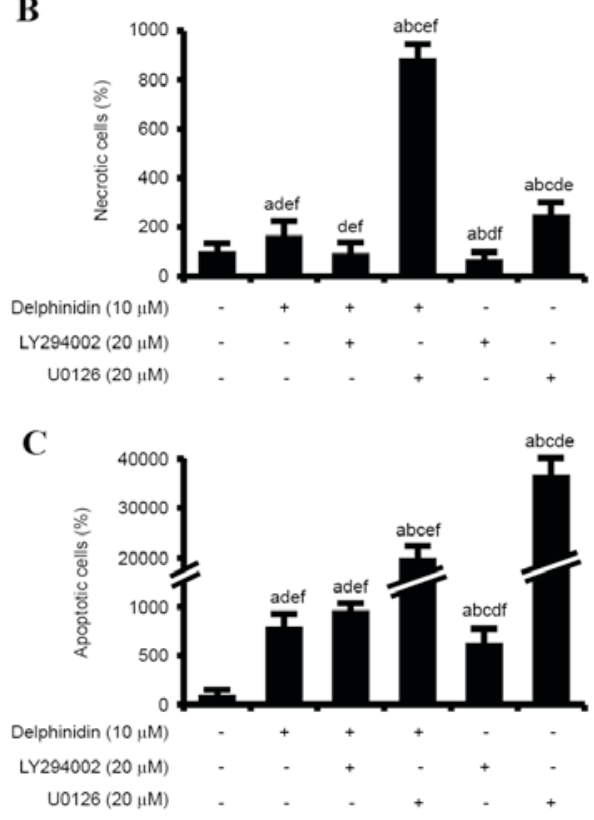

Figure 4. Synergistic effects of delphinidin with pharmacological inhibitors of PI3K/AKT and ERK1/2 MAPK on the necrosis and apoptosis of SKOV3 cells. (A) Flow cytometric analysis of SKOV3 cells by Annexin V and propidium iodide staining. (B) The percentage of cells in the upper left quadrant indicates necrotic cells as compared with non-treated SKOV3 cells. (C) The percentage of cells in the upper right quadrant indicates late apoptotic cells as compared with non-treated SKOV3 cells. Different lowercase letters indicate the statistically significant effects of treatment $(\mathrm{P}<0.05)$ : ${ }^{\mathrm{a}} \mathrm{Compared}$ with non-treated control; ${ }^{\mathrm{b}}$ compared with delphinidin alone; ${ }^{\mathrm{c}}$ compared with delphinidin plus LY294002; ${ }^{\mathrm{d}}$ compared with delphinidin plus U0126; ${ }^{\mathrm{C}}$ compared with LY294002 alone; ${ }^{\mathrm{f}}$ compared with U0126 alone. PI3K, phosphoinositide-3 kinase; ERK, extracellular-regulated kinase; MAPK, mitogen-activated protein kinase; FITC, fluorescein isothiocyanate.

cells, delphinidin inhibited the proliferation of SKOV3 cells by $\sim 40 \%$ ( $\mathrm{P}<0.01)$. Treatment of SKOV3 cells with LY294002 or U0126 alone decreased cell proliferation by $\sim 30 \%(\mathrm{P}<0.05)$ and $70 \%(\mathrm{P}<0.001)$, respectively. In addition, the co-incubation of LY294002 $(\mathrm{P}<0.05)$ and U0126 $(\mathrm{P}<0.001)$ with delphinidin exhibited a synergistic effect to inhibit the proliferation of SKOV3 cells, as compared with delphinidin or pharmacological inhibitor alone. However, no significant effect of SB203580 on the anti-proliferative effects of delphinidin on SKOV3 cells was observed, whereas SB203580 alone slightly reduced the viability of SKOV3 cells. Thus, the expression of target signaling proteins in response to the blockage of PI3K/AKT and ERK1/2 MAPK transduction pathways was investigated using LY294002 or U0126 in order to identify those cell signaling pathways mediated by delphinidin. As illustrated in Fig. 3B, the suppression of AKT in SKOV3 cells was markedly inhibited by combined treatment with LY294002 and delphinidin, and with U0126 and delphinidin, respectively. The levels of phosphorylated ERK1/2 proteins in SKOV3 cells were significantly reduced by administering delphinidin alone $(\mathrm{P}<0.05$; Fig. 3C). In addition, treating cells with a combination of delphinidin with U0126 highly suppressed the abundance of phosphorylated ERK1/2 protein in SKOV3 cells compared with cells treated with delphinidin alone. By contrast, treatment with delphinidin and LY294002 did not result in a significant decrease in the levels of phosphorylated ERK1/2 compared with treatment with dephinidin alone (Fig. 3C). The phosphorylation of P70S6K was also inhibited $(\mathrm{P}<0.05)$ in SKOV3 cells when treated with a combination of LY294002 and delphinidin, but not in cells treated with a combination of U0126 and delphinidin (Fig. 3D). Additionally, pattern of changes in the level of phospho-proteins in response to each treatment in SKOV3 cells were similar for phospho-S6 and phospho-AKT (Fig. 3E). These results indicate that the inactivation of PI3K/AKT and EKR1/2 MAPK signal transduction serves a crucial role in delphinidin-induced suppression of SKOV3 cell proliferation.

Effects of PI3K/AKT and MAPK signal transduction inhibition on the necrosis and apoptosis of SKOV3 cells. In order to compare the apoptotic effects of various drug treatments $(10 \mu \mathrm{M}$ delphinidin, $20 \mu \mathrm{M}$ LY294002, $10 \mu \mathrm{M}$ U0126 and a combination of $10 \mu \mathrm{M}$ delphinidin with each inhibitor) in SKOV3 cells, Annexin V/PI staining and cell cycle assays were performed. As illustrated in Fig. 4A, the number of necrotic (upper left quadrant) and apoptotic (upper right quadrant) SKOV3 cells in response to various drug treatments were investigated by Annexin V-FITC and PI flow cytometry. The number of necrotic SKOV3 cells increased by $165 \%$, as compared with the number of cells in the control cell group (Fig. 4B). In addition, a combination of delphinidin and U0126 increased the abundance of necrotic SKOV3 cells by $\sim 886 \%$, compared with non-treated SKOV3 cells. This necrotic effect on SKOV3 cells was greater than that observed for delphinidin or U0126 treatment alone. However, delphinidin in combination with LY294002 only slightly enhanced the necrosis of SKOV3 cells, as compared with LY294002 administered alone. Delphinidin significantly increased the rate of apoptosis in SKOV3 cells $(793 \%, \mathrm{P}<0.05)$, compared with non-treated SKOV3 cells (Fig. 4C). In addition, the combination of delphinidin and LY294002 increased the number of apoptotic cells to a greater extent than that observed with delphinidin or LY294002 alone. 
A

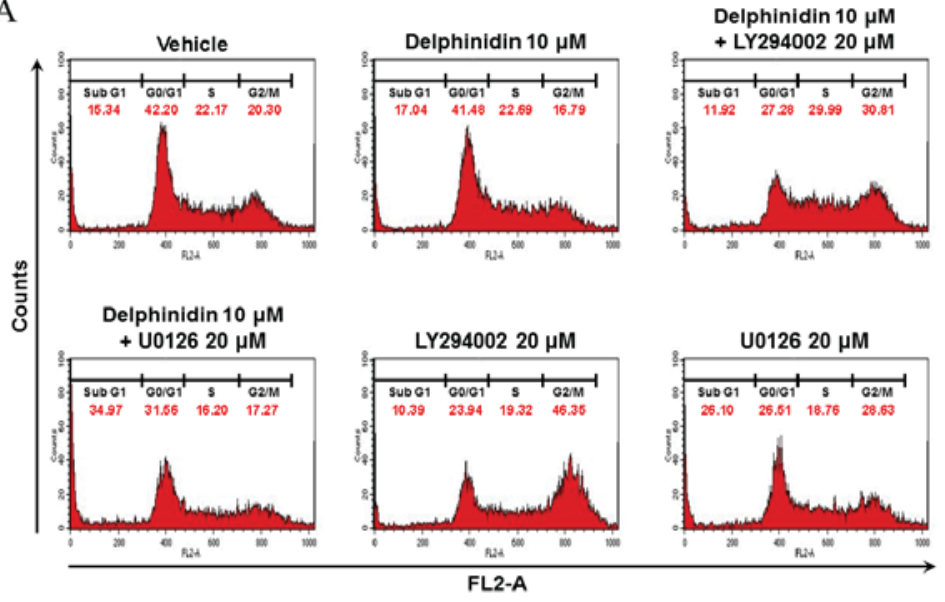

B

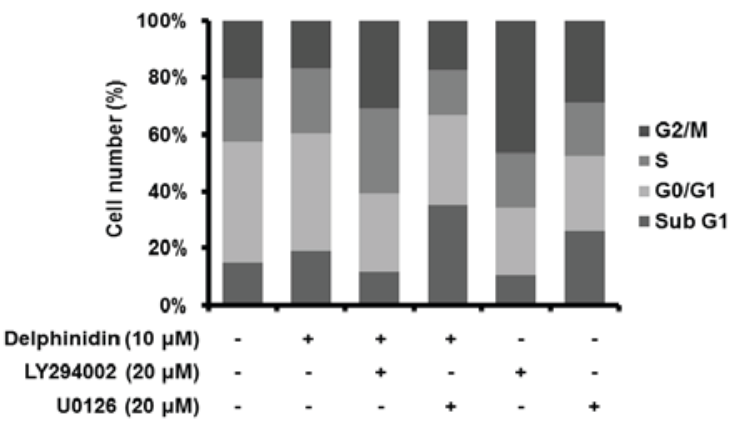

Figure 5. Delphinidin and pharmacological inhibitors regulate the cell cycle of SKOV3 cells. (A) Flow cytometric analysis was used to estimate DNA contents using PI in response to each treatment of SKOV3 cells. (B) The proportion of SKOV3 cells in each stage of the cell cycle was calculated. These results were performed in triplicate.

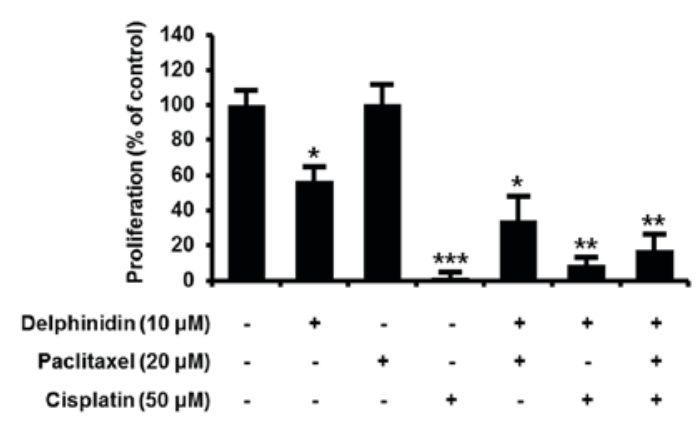

Figure 6. Effects of chemotherapeutic agents with delphinidin on proliferation of SKOV3 cells. Serum starved SKOV3 cells were treated with $10 \mu \mathrm{M}$ delphinidin, $20 \mu \mathrm{M}$ paclitaxel or $50 \mu \mathrm{M}$ cisplatin or their combinations for $48 \mathrm{~h}$. Results indicate relative differences in proliferation with values for non-treated SKOV3 cells set at $100 \%$. ${ }^{* * *} \mathrm{P}<0.001 ;{ }^{* *} \mathrm{P}<0.01 ;{ }^{*} \mathrm{P}<0.05$.

Furthermore, the apoptosis of SKOV3 cells was significantly increased by treatment with U0126. These results indicate that the inhibition of PI3K/AKT and ERK1/2 MAPK signaling pathways in SKOV3 cells treated with delphinidin induces necrosis and apoptosis.

Comparison of cell cycle regulation in SKOV3 cells by delphinidin, inhibitors of PI3K and ERK1/2 MAPK and $a$ combination. To determine the regulatory effects of delphinidin and pharmacological inhibitors on the cell cycle distribution of SKOV3 cells, cell cycle assays were conducted via quantitation of DNA content using PI and flow cytometry (Fig. 5). Delphinidin increased the number of cells in the sub- $\mathrm{G}_{1}$ phase, whereas the proportions of SKOV3 cells in the $G_{0} / G_{1}$ and $G_{2} / M$ phases were decreased following treatment with delphinidin due to cell cycle arrest, compared with non-treated cells. In addition, the treatment of SKOV3 cells with an ERK1/2 MAPK inhibitor (U0126) increased the proportion of cells in the sub- $\mathrm{G}_{1}$ phase compared with non-treated cells. There was a greater increase in the number of SKOV 3 cells in the sub- $G_{1}$ phase when treated with a combination of delphinidin and U0126 compared with treating with delphinidin or U0126 alone. In addition, LY294002 administered alone and in combination with delphinidin increased the proportion of SKOV3 cells in the $\mathrm{S}$ and $\mathrm{G}_{2} / \mathrm{M}$ phases, as compared with $\mathrm{SKOV} 3$ cells treated with delphinidin alone. These results indicate that delphinidin stimulates the apoptosis of SKOV3 cells by inducing cell cycle arrest and that a combination of delphinidin and U0126 primarily induces sub- $\mathrm{G}_{1}$ phase arrest in SKOV3 cells.

Chemotherapeutic effects of delphinidin compared with conventional chemotherapy regimens. To investigate chemotherapeutic effects of delphinidin on the proliferation of SKOV3 cells compared with conventional chemotherapy, the therapeutic efficacy of $10 \mu \mathrm{M}$ delphinidin, $20 \mu \mathrm{M}$ paclitaxel (a taxane-based drug) and $50 \mu \mathrm{M}$ cisplatin (a platinum-based drug) alone and in combination were assessed (Fig. 6). Monotherapy using delphinidin or cisplatin inhibited the proliferation of SKOV3 cells by $44 \%(\mathrm{P}<0.05)$ and $95 \%$ $(\mathrm{P}<0.001)$, respectively. By contrast, the SKOV3 cells were not affected by paclitaxel treatment alone. In comparison with paclitaxel monotherapy, the proliferation of SKOV3 cells was markedly decreased by delphinidin plus paclitaxel. These results indicate that co-treatment with delphinidin and paclitaxel effectively increases the chemotherapeutic activity of paclitaxel monotherapy in SKOV3 cells.

\section{Discussion}

The results of the present study indicate a functional role for delphinidin, a member of the anthocyanidin family, in suppressing the progression of EOC using SKOV3 cells as a model system. The anti-proliferative effects of delphinidin in SKOV3 cells were regulated by its inhibition of PI3K/AKT and ERK1/2 MAPK signal transduction protein phosphorylation. In addition, a combination of delphinidin and the pharmacological inhibitors of PI3K and ERK1/2 MAPK induced apoptosis and cell cycle arrest in SKOV3 cells. Furthermore, comparative analyses between delphinidin and conventional chemotherapy of EOC revealed that delphinidin exhibits a synergistic effect with paclitaxel in promoting anti-proliferative effects in SKOV3 cells. These findings support the 
hypothesis that delphinidin inhibits cell proliferation and induces apoptosis in EOC, and that appropriate therapeutic approaches using delphinidin may be useful for overcoming chemoresistance in ovarian cancer cells.

Delphinidin, a natural bioactive product belonging to the anthocyanidins, has a diphenylpropane-based polyphenolic ring structure, including a positive charge in its core ring (21). The clinical interest in delphinidin is increasing due to its potential in preventing aging, cardiovascular diseases, neurodegenerative diseases, diabetes and cancer through various antioxidative (7), anti-inflammatory (22), anti-angiogenic (23) and anti-mutagenic (24) properties. In particular, the antiproliferative effects of delphinidin have been identified in various types of cancer cells to involve the regulation of intracellular signaling pathways. In hepatocellular carcinoma, delphinidin induces apoptotic cell death through the induction of B-cell lymphoma-2 (Bcl-2) associated $\mathrm{X}$ protein and the reduction of Bcl-2 (25). Delphinidin also sensitizes TNF-related apoptosis-inducing ligand-resistant prostate cancer cells to undergo apoptosis by inducing death receptor 5 mediating histone deacetylase 3 cleavage (9). In addition, it suppresses the progression of lung cancer by inhibiting the aberrant expression of endothelial growth factor receptor and vascular endothelial growth factor receptor 2, leading to the decreased phosphorylation of PI3K and MAPK (10). In addition, delphinidin blocks the phosphorylation of ERK1/2 MAPK in breast cancer cells (8). However, little is known about the function and underlying mechanisms of delphinidin in EOC. The present study demonstrated that the antiproliferative effects of delphinidin on SKOV3 cells are dose-dependent (Fig. 1A). In addition, delphinidin increased the percentage of apoptotic SKOV3 cells. These results indicate that delphinidin inhibits the proliferation and induces the apoptosis of SKOV3 cells.

EOC initiation and progression are complex events regulated by various intracellular signaling proteins (26). For a novel therapeutic strategy to incorporate delphinidin as an anticancer agent, understanding of the molecular mechanisms underlying the process of carcinogenesis from ovarian surface epithelia is essential. Activation of the PI3K/AKT pathway is considered a key signal transduction pathway for preventing tumorigenesis through promoting cell survival and proliferation, and blocking apoptosis via the activation of pro-apoptotic proteins and the control of cell cycle regulatory proteins $(27,28)$. Activated AKT may stimulate phosphorylation of mechanistic target of rapamycin (mTOR) or directly phosphorylate p70 S6 kinase (P70S6K), regulating translation through ribosomal protein S6 (S6) for cell proliferation and survival (29). The activation of P70S6K in cell proliferation, invasiveness and angiogenesis in malignant ovarian tumors has been reported and is recognized as a hallmark of ovarian cancer $(30,31)$. The aberrant alteration of the PI3K-AKT-mTOR signaling pathways indicates that the control of target proteins in the PI3K-AKT-mTOR signal transduction pathway may be regarded as an effective therapeutic approach for the treatment of EOC (32). Consistent with the results of previous studies, the data obtained in the current study indicate that increasing concentrations of delphinidin were able to decrease the activity of AKT in SKOV3 cells. In addition, the phosphorylation of P70S6K and S6 was inactivated following the treatment of SKOV3 cells with delphinidin. Delphinidin administered in combination with LY294002 suppressed the activity of phosphorylated AKT and S6 proteins, as compared with delphinidin monotherapy in SKOV3 cells, and enhanced the antiproliferative effects of delphinidin in SKOV3 cells. These results indicate that delphinidin exerts chemopreventive effects on EOC cells through the inhibition of PI3K/AKT signaling pathways.

Constitutive activation of the ERK1/2 MAPK pathway promotes the growth, proliferation and differentiation of ovarian cancer cells via the activation of downstream protein kinase or transcription factors that may enhance carcinogenesis $(33,34)$. ERK1/2 MAPK signaling is activated by mitogenic factors and inhibition of this pathway is crucial for the treatment of EOC $(34,35)$. In the present study, delphinidin downregulated the phosphorylation of ERK1/2 MAPK in SKOV3 cells in a dose-dependent manner. UO126, a pharmacological inhibitor of ERK1/2, completely blocked the phosphorylation of ERK1/2 in SKOV3 cells, whereas LY294002 did not inhibit the phosphorylation of ERK1/2. In addition, the combination of U0126 and delphinidin enhanced the antiproliferative effects on SKOV3 cells, as compared with delphinidin or U0126 alone. This effective blockade of signal transduction using an ERK1/2 MAPK inhibitor and delphinidin may provide insight into potential strategies for preventing carcinogenesis from the ovarian surface epithelium.

For identifying interactions between delphinidin and the pharmacological inhibitors of PI3K and ERK1/2 MAPK signaling, apoptosis and cell cycle regulation in response to a combination of delphinidin with LY294002 or U0126 was investigated. As demonstrated in previous studies, delphinidin induces cell cycle arrest via the downregulation of certain cell cycle regulatory proteins, including cyclin-dependent kinase (Cdk)1, Cdk2, cyclin A and cyclin D1, and via the upregulation of $\mathrm{p} 21^{\mathrm{WAF} 1}$ in a dose-dependent manner in prostate and colon cancer cells $(12,36)$. In addition, PI3K/AKT and ERK1/2-mediated suppression of cell proliferation is due to cell cycle arrest in various types of cancer cells (37-41). Concordantly, the results of the present study revealed that the combination of delphinidin and U0126 increased the percentage of necrotic cells, as compared with the effects of delphinidin or U0126 alone. In addition, a combination of delphinidin with LY294002 or U0126 synergistically increased the rate of apoptosis in SKOV3 cells, as compared with the effects of delphinidin alone. According to the cell cycle analysis, the apoptotic effects were regulated through cell cycle arrest at the sub- $\mathrm{G}_{1}$ phase. The cooperative effects of delphinidin and the inhibitors of PI3K/AKT and ERK1/2 MAPK induced the apoptosis of SKOV3 cells via regulation of the cell cycle.

Despite initial positive responses to platinum- and taxane-based chemotherapies, persistent chemoresistance of EOC is consistently a problem in $\leq 80 \%$ of patients (17). Specifically, the continued exposure of SKOV3 cells to paclitaxel results in chemoresistance with marked morphological changes $(42,43)$. Therefore, novel therapeutic strategies for the treatment of EOC are required for improvement of progression-free survival and overall survival in patients. The results of the present study indicated that the treatment of SKOV3 cells with paclitaxel for $48 \mathrm{~h}$ did not affect the viability of SKOV3 cells, whereas cisplatin significantly decreased the 
proliferation of SKOV3 cells. However, delphinidin exhibited synergistic anticancer properties by reducing the proliferation of paclitaxel-resistant SKOV3 cells. Therefore, combination therapy with delphinidin and paclitaxel represents a novel treatment strategy for additional clinical studies into advanced stages of EOC.

Collectively, the present study presents evidence, using the SKOV3 human ovarian cancer cell line, that delphinidin is a crucial physiological modulator in EOC, and is also an inhibitor of EOC cell proliferation. It was identified that delphinidin increased the rate of apoptosis via DNA fragmentation in SKOV3 cells. In addition, delphinidin suppressed the proliferation of SKOV3 cells through inactivating the phosphorylation of AKT, P70S6K, S6 and ERK1/2 proteins in the PI3K/AKT and ERK1/2 MAPK signaling pathways. Furthermore, blocking the ERK1/2 MAPK signaling pathways reduced the levels of S6 protein in SKOV3 cells, which regulates protein synthesis for cell death. Furthermore, interactions between delphinidin and various pharmacological inhibitors of PI3K/AKT and ERK1/2 MAPK, synergistically induced apoptosis by increasing the proportion of SKOV3 cells arrested in the sub- $\mathrm{G}_{1}$ phase of the cell cycle. Delphinidin-reduced proliferation of SKOV3 cells was also required for paclitaxel to decrease the proliferation of SKOV3 cells. In conclusion, these findings provide evidence for the synergistic anticancer effects of delphinidin combined with paclitaxel in human ovarian cancer SKOV3 cells, involving the inactivation of the PI3K/AKT and ERK1/2 MAPK signaling pathways. Thus, delphinidin may be developed as a novel therapeutic agent for the enhancement of survival in patients with EOC.

\section{Acknowledgements}

The authors thank Dr Fuller W. Bazer (Texas A\&M University, College Station, USA) for their thoughtful editing and comments on this manuscript. The present study was supported by grants from the Basic Science Research Program (grant no. 2015R1D1A1A01059331) of the Republic of Korea and also supported by School of Life Sciences and Biotechnology for BK21 PLUS, Korea University.

\section{References}

1. Ververidis F, Trantas E, Douglas C, Vollmer G, Kretzschmar G and Panopoulos N: Biotechnology of flavonoids and other phenylpropanoid-derived natural products. Part I: Chemical diversity, impacts on plant biology and human health. Biotechnol J 2: 1214-1234, 2007.

2. Zamora-Ros R, Touillaud M, Rothwell JA, Romieu I and Scalbert A: Measuring exposure to the polyphenol metabolome in observational epidemiologic studies: Current tools and applications and their limits. Am J Clin Nutr 100: 11-26, 2014.

3. Prior RL and Wu X: Anthocyanins: Structural characteristics that result in unique metabolic patterns and biological activities. Free Radic Res 40: 1014-1028, 2006.

4. Mazza G and Miniati E: Anthocyanins in fruits, vegetables, and grains. CRC Press, Boca Raton, 1993.

5. Favot L, Martin S, Keravis T, Andriantsitohaina R and Lugnier C: Involvement of cyclin-dependent pathway in the inhibitory effect of delphinidin on angiogenesis. Cardiovasc Res 59: 479-487, 2003.

6. Lamy S, Lafleur R, Bédard V, Moghrabi A, Barrette S, Gingras D and Béliveau R: Anthocyanidins inhibit migration of glioblastoma cells: Structure-activity relationship and involvement of the plasminolytic system. J Cell Biochem 100: 100-111, 2007.
7. Noda Y, Kaneyuki T, Mori A and Packer L: Antioxidant activities of pomegranate fruit extract and its anthocyanidins: Delphinidin, cyanidin, and pelargonidin. J Agric Food Chem 50: 166-171, 2002.

8. Ozbay T and Nahta R: Delphinidin inhibits HER2 and Erk1/2 signaling and suppresses growth of HER2-overexpressing and triple negative breast cancer cell lines. Breast cancer (Auckl) 5: 143-154, 2011.

9. Ko H, Jeong MH, Jeon H, Sung GJ, So Y, Kim I, Son J, Lee SW, Yoon HG and Choi KC: Delphinidin sensitizes prostate cancer cells to TRAIL-induced apoptosis, by inducing DR5 and causing caspase-mediated HDAC3 cleavage. Oncotarget 6: 9970-9984, 2015.

10. Pal HC, Sharma S, Strickland LR, Agarwal J, Athar M, Elmets CA and Afaq F: Delphinidin reduces cell proliferation and induces apoptosis of non-small-cell lung cancer cells by targeting EGFR/VEGFR2 signaling pathways. PLoS One 8: e77270, 2013.

11. Feng R, Wang SY, Shi YH, Fan J and Yin XM: Delphinidin induces necrosis in hepatocellular carcinoma cells in the presence of 3-methyladenine, an autophagy inhibitor. J Agric Food Chem 58: 3957-3964, 2010.

12. Yun JM, Afaq F, Khan N and Mukhtar H: Delphinidin, an anthocyanidin in pigmented fruits and vegetables, induces apoptosis and cell cycle arrest in human colon cancer HCT116 cells. Mol Carcinog 48: 260-270, 2009.

13. Filipiak K, Hidalgo M, Silvan JM, Fabre B, Carbajo RJ, Pineda-Lucena A, Ramos A, de Pascual-Teresa B and de Pascual-Teresa S: Dietary gallic acid and anthocyanin cytotoxicity on human fibrosarcoma HT1080 cells. A study on the mode of action. Food Funct 5: 381-389, 2014.

14. de Hullu JA, van der Avoort IA, Oonk MH and van der Zee AG: Management of vulvar cancers. Eur J Surg Oncol 32: 825-831, 2006.

15. Kochli OR, Sevin BU, Averette HE and Haller U: Overview of currently used chemosensitivity test systems in gynecologic malignancies and breast cancer. Contrib Gynecol Obstet 19: 12-23, 1994.

16. Siegel RL, Miller KD and Jemal A: Cancer statistics, 2015. CA Cancer J Clin 65: 5-29, 2015.

17. Raja FA, Chopra N and Ledermann JA: Optimal first-line treatment in ovarian cancer. Ann Oncol 23 (Suppl 10): x118-x127, 2012.

18. Javadi S, Ganeshan DM, Qayyum A, Iyer RB and Bhosale P: Ovarian cancer, the revised FIGO staging system and the role of imaging. AJR Am J Roentgenol 206: 1351-1360, 2016.

19. Coleman RL, Monk BJ, Sood AK and Herzog TJ: Latest research and treatment of advanced-stage epithelial ovarian cancer. Nat Rev Clin Oncol 10: 211-224, 2013.

20. Tripathi V, Kumar R, Dinda AK, Kaur J and Luthra K: CXCL12-CXCR7 signaling activates ERK and Akt pathways in human choriocarcinoma cells. Cell Commun Adhes 21: 221-228, 2014.

21. Hou DX, Fujii M, Terahara N and Yoshimoto M: Molecular mechanisms behind the chemopreventive effects of anthocyanidins. J Biomed Biotechnol 2004: 321-325, 2004.

22. Hou DX, Yanagita T, Uto T, Masuzaki S and Fujii M: Anthocyanidins inhibit cyclooxygenase-2 expression in LPS-evoked macrophages: Structure-activity relationship and molecular mechanisms involved. Biochemical pharmacology 70: 417-425, 2005.

23. Lamy S, Blanchette M, Michaud-Levesque J, Lafleur R, Durocher Y, Moghrabi A, Barrette S, Gingras D and Béliveau R: Delphinidin, a dietary anthocyanidin, inhibits vascular endothelial growth factor receptor-2 phosphorylation. Carcinogenesis 27: 989-996, 2006

24. Azevedo L, Alves de Lima PL, Gomes JC, Stringheta PC, Ribeiro DA and Salvadori DMF: Differential response related to genotoxicity between eggplant (Solanum melanogena) skin aqueous extract and its main purified anthocyanin (delphinidin) in vivo. Food Chem Toxicol 45: 852-858, 2007.

25. Yeh CT and Yen GC: Induction of apoptosis by the Anthocyanidins through regulation of Bcl-2 gene and activation of c-Jun N-terminal kinase cascade in hepatoma cells. J Agric Food Chem 53: 1740-1749, 2005.

26. Smolle E, Taucher V, Pichler M, Petru E, Lax S and Haybaeck J: Targeting signaling pathways in epithelial ovarian cancer. Int $J$ Mol Sci 14: 9536-9555, 2013.

27. Luo J, Manning BD and Cantley LC: Targeting the PI3K-Akt pathway in human cancer: Rationale and promise. Cancer Cell 4: 257-262, 2003. 
28. Osaki M, Oshimura M and Ito H: PI3K-Akt pathway: Its functions and alterations in human cancer. Apoptosis 9: 667-676, 2004.

29. Ip CKM and Wong AST: Exploiting p70 S6 kinase as a target for ovarian cancer. Expert Opin Ther Tar 16: 619-630, 2012.

30. Meng Q, Xia C, Fang J, Rojanasakul Y and Jiang BH: Role of $\mathrm{PI} 3 \mathrm{~K}$ and AKT specific isoforms in ovarian cancer cell migration, invasion and proliferation through the p70S6K1 pathway. Cell Signal 18: 2262-2271, 2006.

31. Pon YL, Zhou HY, Cheung AN, Ngan HY and Wong AS: p70 S6 kinase promotes epithelial to mesenchymal transition through snail induction in ovarian cancer cells. Cancer Res 68 6524-6532, 2008.

32. Cheaib B, Auguste A and Leary A: The PI3K/Akt/mTOR pathway in ovarian cancer: Therapeutic opportunities and challenges. Chin J Cancer 34: 4-16, 2015.

33. Choi KC, Auersperg N and Leung PC: Mitogen-activated protein kinases in normal and (pre)neoplastic ovarian surface epithelium. Reprod Biol Endocrinol 1: 71, 2003.

34. Roberts PJ and Der CJ: Targeting the Raf-MEK-ERK mitogen-activated protein kinase cascade for the treatment of cancer. Oncogene 26: 3291-3310, 2007.

35. Nicosia SV, Bai W, Cheng JQ, Coppola D and Kruk PA: Oncogenic pathways implicated in ovarian epithelial cancer. Hemato Oncol Clin North Am 17: 927-943, 2003.

36. Bin Hafeez B, Asim M, Siddiqui IA, Adhami VM, Murtaza I and Mukhtar H: Delphinidin, a dietary anthocyanidin in pigmented fruits and vegetables: A new weapon to blunt prostate cancer growth. Cell Cycle 7: 3320-3326, 2008.
37. Fan W, Sun L, Zhou JQ, Zhang C, Qin S, Tang Y, Liu Y, Lin SS and Yuan ST: Marsdenia tenacissima extract induces G0/G1 cell cycle arrest in human esophageal carcinoma cells by inhibiting mitogen-activated protein kinase (MAPK) signaling pathway. Chin J Nat Med 13: 428-437, 2015.

38. Li A, Wang J, Wu M, Zhang X and Zhang $\mathrm{H}$ : The inhibition of activated hepatic stellate cells proliferation by arctigenin through G0/G1 phase cell cycle arrest: Persistent p27(Kip1) induction by interfering with PI3K/Akt/FOXO3a signaling pathway. Eur J Pharmacol 747: 71-87, 2015.

39. Lin CJ, Chen TL, Tseng YY, Wu GJ, Hsieh MH, Lin YW and Chen RM: Honokiol induces autophagic cell death in malignant glioma through reactive oxygen species-mediated regulation of the p53/PI3K/Akt/mTOR signaling pathway. Toxicol Appl Pharmacol 304: 59-69, 2016

40. Liu E, Li J, Shi S, Wang X, Liang T, Wu B and Li Q: Sustained ERK activation-mediated proliferation inhibition of farrerol on human gastric carcinoma cell line by G0/G1-phase cell-cycle arrest. Eur J Cancer Prev 25: 490-499, 2016.

41. Oh I, Cho H, Lee Y, Cheon M, Park D and Lee Y: Blockage of autophagy rescues the dual PI3K/mTOR inhibitor BEZ235-induced growth inhibition of colorectal cancer cells. Dev Reprod 20: 1-10, 2016.

42. Jia L, Zhang S, Ye Y, Li X, Mercado-Uribe I, Bast RC Jr and Liu J: Paclitaxel inhibits ovarian tumor growth by inducing epithelial cancer cells to benign fibroblast-like cells. Cancer Lett 326: 176-182, 2012.

43. Lamendola DE, Duan Z, Yusuf RZ and Seiden MV: Molecular description of evolving paclitaxel resistance in the SKOV-3 human ovarian carcinoma cell line. Cancer Res 63: 2200-2205, 2003. 